\title{
Neutrophil-to-Lymphocyte Ratio: Can It Be Used as an Adjunct Tool to Predict Histopathological Grade of Brain Tumor?
}

\author{
K. G. Ashwath ${ }^{1} \quad$ Ashish Aggarwal $^{1} \quad$ Kokkula Praneeth ${ }^{1} \quad$ Navneet Singla ${ }^{1} \quad$ Kirti Gupta ${ }^{2}$ \\ ${ }^{1}$ Department of Neurosurgery, Post Graduate Institute of Medical \\ Education and Research, Chandigarh, India \\ 2Department of Pathology, Post Graduate Institute of Medical \\ Education and Research, Chandigarh, India

\begin{abstract}
Address for correspondence Ashish Aggarwal, MCh Neurosurgery, Department of Neurosurgery, Post Graduate Institute of Medical Education and Research, Chandigarh 160 012, India (e-mail: aaggarwal_7@yahoo.com).
\end{abstract}

J Neurosci Rural Pract 2019;10:648-652

\begin{abstract}
Background Since histopathology is available only after surgery, clinical condition and radiological characters of the tumor are important factors on which a clinician counsels the patient of brain tumor to take a decision regarding the management. Neutrophil lymphocyte ratio (NLR), a marker of inflammation can be used as a prognostic marker to predict the survival in high-grade gliomas and metastases. We evaluated the utility of NLR as an adjunct tool in predicting the histopathological grade of brain tumors.

Materials and Methods One hundred sixteen patients with a diagnosis of brain tumors planned for surgical excision or biopsy were enrolled in the study. NLR was estimated in the preoperative blood sample. Patients were grouped into low- and highgrade brain tumors and their mean NLRs were analyzed. Similar evaluation was carried out between the intra- and extra-axial tumors.

Results Mean age of the study group was 40.14 years with 61 males. Seventy-eight patients had low-grade tumor and 38 patients had high-grade tumor. Sixty patients had extra-axial tumors and 56 patients had intra-axial tumors. The mean NLR of lowgrade tumors was $1.68 \pm 0.53$ and that of high-grade tumors was $3.12 \pm 0.74$. NLR $>2.4$ can be used to identify high-grade brain tumors with a sensitivity of $80 \%$, specificity

Keywords

- brain tumors

- histopathological grade

- location of tumor

- neutrophil-to-lymphocyte ratio of $92 \%$, positive predictive value of $82.1 \%$, negative predictive value of $91 \%$, an excellent impact with likelihood ratio (+) of 10.1 , and an odds ratio of 54.1. The mean NLR of extra-axial tumors was $1.68+0.62$ and that of intra-axial tumors was $2.64 \pm 0.91$. These observations were statistically significant with $p$-value $<0.05$.

Conclusions NLR is an easily available and inexpensive marker of systemic inflammation, which varies across different histopathological grades of brain tumors. Mean NLR is higher in high-grade tumors and also intra-axial tumors with a cutoff value of NLR $>2.4$ and $>2.0$, respectively.
\end{abstract}

\section{Introduction}

The average survival in cases of glioblastoma multiforme remains dismal in spite of continuous sustained efforts to understand the disease pathophysiology. This is in addition to tremendous morbidity and psychological stress to caregivers. Once diagnosed, this remains a sort of death sentence, the only question is when? On the other hand, there are neoplasms that are benign, and once successfully excised, a patient can expect a near-normal life span. With such a wide spectrum of brain tumors, it becomes imperative to prognosticate the disease at the onset so that the appropriate management decisions can be taken in consonance with the wishes of the patient and family members.
DOI https://doi.org/ $10.1055 / \mathrm{s}-0039-3399489$ ISSN 0976-3147.
C)2019 Association for Helping Neurosurgical Sick People
License terms

(요 (1) $\odot \circledast$ 
Although radiology provides us some answers, the exact nature of tumor and the prognosis can be elucidated only after detailed histopathology. This takes time and is available only after surgery. Is there a method whereby neurosurgeons can be wiser in the preoperative period regarding nature of tumor and consequently its prognosis?

There is an increasing and consistent evidence that cancer-associated inflammation and accumulation of inflammatory cells around cancer cells is a key determinant of outcome in patients with cancer. ${ }^{1-8} \mathrm{~A}$ systematic inflammatory response secondary to cancer leads to changes in the relative levels of circulating white blood cells, and with the progression of cancer, this results in an increase in neutrophils and a decrease in lymphocytes. ${ }^{2}$ Among the various inflammatory markers, neutrophil-to-lymphocyte ratio (NLR) is one of the readily available markers and has been proved to be a prognostic marker to predict the survival in high-grade glioma and metastases. ${ }^{9-21}$

In the present study, we assessed the utility of NLR as an adjunct tool in predicting the histopathological grade of brain tumor and indirectly the prognosis.

\section{Materials and Methods}

One hundred and sixteen patients with a preoperative diagnosis of brain tumor, who were planned for surgery, were enrolled in the study. Detailed clinical examination and radiological evaluation were performed. NLR was calculated in the preoperative blood sample. After the surgery, histopathology of the excised sample was studied and compared with preoperative NLR. Following patients were excluded from the study-patients undergoing redo surgery and any evidence of inflammation (such as diabetes mellitus, metabolic syndrome, heart disease, severe renal or hepatic dysfunction, other cancers, inflammatory diseases, and history of previous infection in the preceding 3 months) and patients on anti-inflammatory drugs including steroids.

Brain tumors were classified according to the World Health Organization (WHO) classification and grading of central nervous system tumors and categorized under two groups-low- and high-grade tumors. ${ }^{22}$ Low-grade tumors include mainly Grade I meningioma, schwannoma, colloid cyst, craniopharyngioma, dermoid and epidermoid, Grade I to II gliomas, oligodendroglioma, ependymoma, and choroid plexus papilloma. High-grade tumors included Grade II to III meningiomas, Grade III to IV gliomas, anaplastic ependymoma, medulloblastoma, anaplastic oligodendroglioma, metastases, lymphoma, and choroid plexus carcinoma. Depending on the origin and location, the tumors were classified as extra- and intra-axial.

\section{Statistics}

Patients were grouped into low- and high-grade brain tumors, and their NLR was analyzed using SPSS version 23 software (IBM Corp, New York, United States). Groups were compared using chi-squared test and independent sample test after confirming the normality of distribution.
Two-sided significance test was used, and the significance was kept at $p<0.05$. Receiver operator characteristic (ROC) curve was used to determine the cutoff for NLR to predict grade of tumor. Sensitivity, specificity, positive predictive value, negative predictive value, likelihood ratio, and odds ratio based on the NLR cutoff estimated from ROC curve were calculated. Similar evaluation was performed between the intra- and extra-axial tumors with subgroup analysis.

\section{Results}

The mean age of the study group was 40.14 (range: 6-77 years) years with 61 males and 55 females. Seventy-eight patients had low-grade tumor and 38 had high-grade tumor. Sixty patients had extra-axial tumors and 56 had intra-axial tumors. Extra-axial tumors included 52 low-grade lesions and 8 high-grade lesions (Grade II-III meningiomas). Intra-axial tumors included 26 low-grade tumors (WHO Grades I and II) and 30 high-grade tumors (WHO Grades III and IV) (-Table 1).

The mean NLR of low-grade tumors was $1.68 \pm 0.53$ and that of high-grade tumors was $3.12 \pm 0.74$ ( $\boldsymbol{- F i g .} \mathbf{1}$ ). This was statistically significant $(p<0.01)$. ROC curve for grade of tumor and NLR shows significant area under the curve (AUC) of 0.94 and NLR cutoff at 2.4 (-Fig. 2).

The highest values of mean NLR were found in two of the most aggressive tumors, namely glioblastoma multiforme (3.17) and brain metastasis (3.32).

A cutoff value of NLR >2.4 can be used to predict highgrade brain tumors with a sensitivity of $80 \%$, specificity of $92 \%$, positive predictive value of $82.1 \%$, negative predictive value of $91 \%$, an excellent impact with likelihood ratio (+) of 10.1 , and an odds ratio of 54.1.

ROC curve for the location of tumor and NLR shows AUC of 0.71 ( - Fig. 3). A cutoff value of NLR $>2$ can be used to predict intra-axial brain tumors with a sensitivity of $75 \%$ and specificity of $61.7 \%$.

In subgroup analysis, of extra-axial tumors, NLR of highgrade tumor $(2.71 \pm 0.73)$ was higher than that of low-grade tumors $(1.53 \pm 0.43)$. Of intra-axial tumors, NLR of highgrade tumor $(3.23 \pm 0.71)$ was higher than that of low-grade tumor $(1.97 \pm 0.58)$. These observations were statistically significant with $p<0.05$. This shows that grade of tumor would affect the NLR even within the subgroups of extra- and intra-axial tumors.

Among meningiomas, Grade 1 meningiomas had a lower mean NLR (1.46) than Grade II and III meningiomas (mean $\mathrm{NLR}=2.77)$.

\section{Discussion}

Histopathological grade of brain tumor is the most important factor determining the outcome of the patient. Malignant brain tumors, especially glioblastoma multiforme and metastasis, continue to have unacceptably low overall survival. Although radiological characteristics give an idea about the grade of the tumor, exact prognostication depends on 
Table 1 Relation of each tumor type with neutrophil-to-lymphocyte ratio, grade, and axiality

\begin{tabular}{|c|c|c|c|c|c|c|}
\hline Types of tumor & $\begin{array}{l}\text { Nature of } \\
\text { tumor }\end{array}$ & $\begin{array}{l}\text { Axiality of } \\
\text { tumor }\end{array}$ & $\begin{array}{l}\text { Number of } \\
\text { patients }\end{array}$ & $\begin{array}{l}\text { Mean } \\
\text { NLR }\end{array}$ & $\begin{array}{l}\text { Whether } \\
\text { follows cutoff } \\
\text { value for the } \\
\text { grade }\end{array}$ & $\begin{array}{l}\text { Whether follows } \\
\text { cutoff value for } \\
\text { axiality }\end{array}$ \\
\hline Craniopharyngioma & LG & EA & 3 & 1.59 & Yes & Yes \\
\hline Schwannoma & LG & EA & 8 & 1.93 & Yes & Yes \\
\hline Dermoid cyst & LG & EA & 2 & 1.31 & Yes & Yes \\
\hline Epidermoid cyst & LG & EA & 4 & 1.23 & Yes & Yes \\
\hline Transitional meningioma & LG & EA & 4 & 1.65 & Yes & Yes \\
\hline Psammomatous meningioma & LG & EA & 2 & 1.58 & Yes & Yes \\
\hline Meningothelial meningioma & LG & EA & 29 & 1.47 & Yes & Yes \\
\hline $\begin{array}{l}\text { Lymphoplasmacytic } \\
\text { meningioma }\end{array}$ & $\mathrm{HG}$ & EA & 1 & 2.72 & Yes & No \\
\hline Hemangiopericytoma & HG & EA & 1 & 2.23 & No & No \\
\hline Atypical meningioma & HG & EA & 5 & 2.47 & Yes & No \\
\hline Anaplastic meningioma & $\mathrm{HG}$ & EA & 1 & 4.34 & Yes & No \\
\hline Angiocentric glioma & LG & IA & 2 & 2.24 & Yes & Yes \\
\hline Choroid plexus papilloma & LG & IA & 1 & 1.71 & Yes & No \\
\hline Colloid cyst & LG & IA & 3 & 1.28 & Yes & No \\
\hline Germinocystic astrocytoma & LG & IA & 1 & 1.58 & Yes & No \\
\hline Hemangioblastoma & LG & IA & 2 & 1.21 & Yes & No \\
\hline Pilocytic astrocytoma & LG & IA & 10 & 1.90 & Yes & No \\
\hline Central neurocytoma & LG & IA & 1 & 3.18 & No & Yes \\
\hline Ependymoma & LG & IA & 2 & 3.05 & No & Yes \\
\hline Oligodendroglioma & LG & IA & 4 & 2.41 & No & Yes \\
\hline Anaplastic astrocytoma & $\mathrm{HG}$ & IA & 3 & 2.53 & Yes & Yes \\
\hline Anaplastic oligodendroglioma & HG & IA & 1 & 2.91 & Yes & Yes \\
\hline Medulloblastoma & HG & IA & 3 & 2.93 & Yes & Yes \\
\hline Glioblastoma multiforme & $\mathrm{HG}$ & IA & 11 & 3.39 & Yes & Yes \\
\hline Diffuse large B cell lymphoma & HG & IA & 1 & 3.7 & Yes & Yes \\
\hline Metastases & $\mathrm{HG}$ & IA & 11 & 3.32 & Yes & Yes \\
\hline
\end{tabular}

Abbreviations: EA, extra-axial; HG, high grade; IA, intra-axial; LG, low grade; NLR, neutrophil-to-lymphocyte ratio.

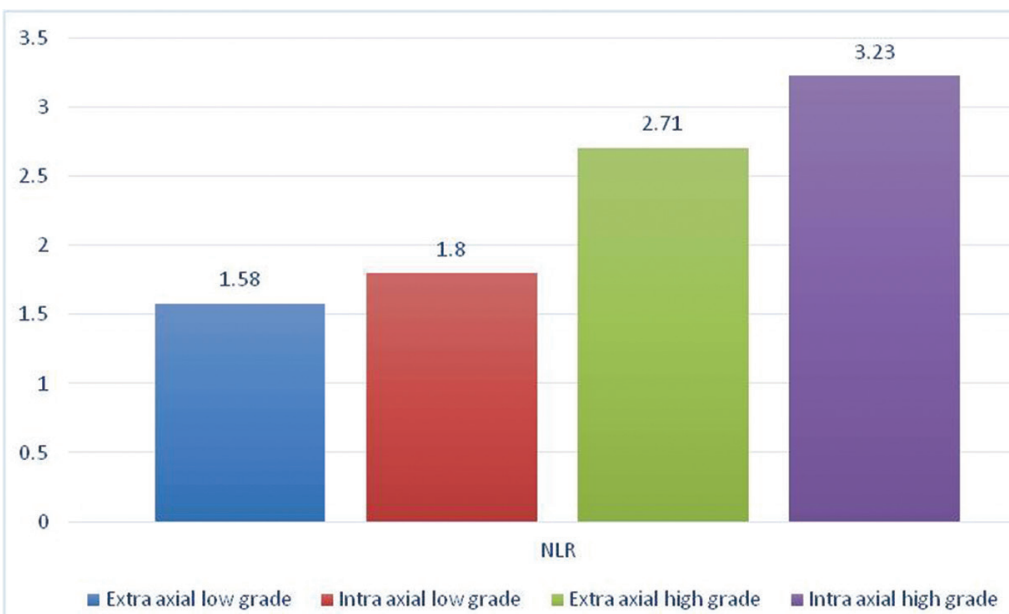

Fig. 1 Mean neutrophil-to-lymphocyte ratio of different grades and locations of brain tumors, relation of each tumor type with neutrophil-to-lymphocyte ratio, grade, and axiality. ROC, receiver operator characteristic. 


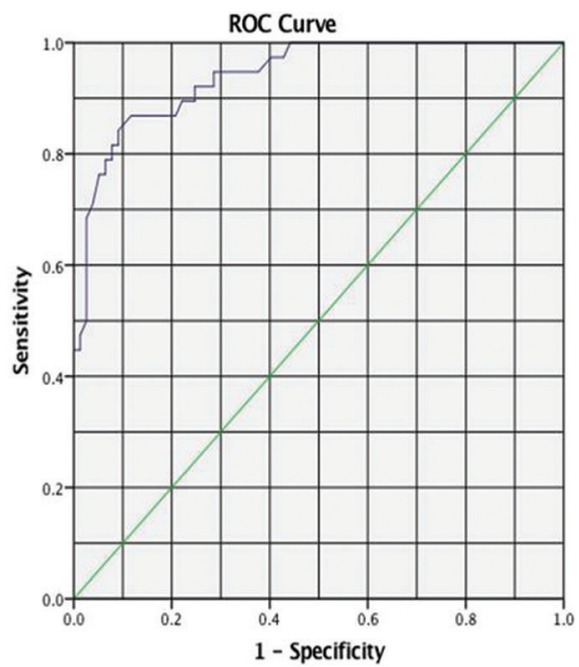

Fig. 2 Receiver operator characteristic curve for neutrophil-to-lymphocyte ratio (NLR) and grade of tumor.

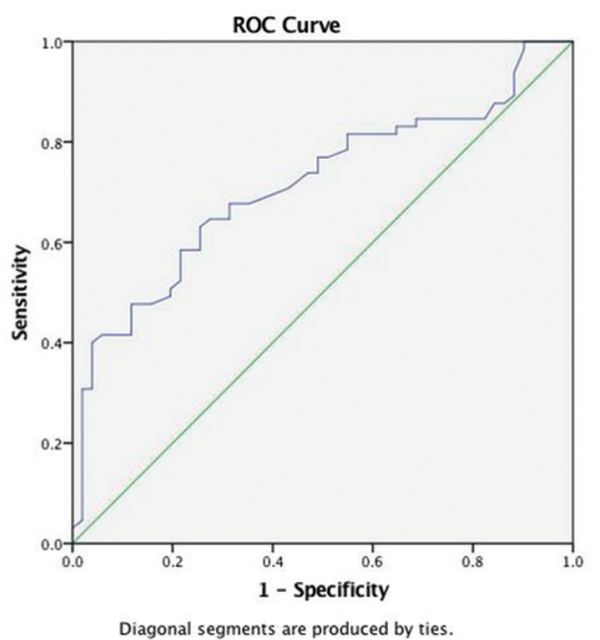

Fig. 3 Receiver operator characteristic (ROC) curve for neutrophil-to-lymphocyte ratio and location of tumor.

histopathology of the tumor. This is available only in the postoperative period.

Can there be a method by which we can in the preoperative period have some clues as to the nature of tumor?

In recent times, there has been an increasing interest in the role of inflammation in malignancy. ${ }^{1-8}$ Several studies have established the occurrence of a systemic inflammatory response in malignancies. The degree and type of inflammatory response depend on the antigenicity and vascularity of the lesion. Thus, it may reasonably be expected that the degree of derangement of systemic markers of inflammation varies, based on biologic aggressiveness of the neoplasm.

Recruitment of more neutrophils as compared with lymphocytes around cancer cells portends a worse prognosis. ${ }^{2}$ This reflects not only in the peritumoral tissue but also in the peripheral blood, thus altering NLR. NLR has shown to have prognostic value in several cancers including lung, breast, and kidney cancers. ${ }^{1,4-8}$ We explored the possibility of using preoperative NLR as an adjunct to radiology to distinguish between high- and low-grade tumors to help carry out prognostication in the preoperative period.

We studied 116 patients of brain tumor covering the whole spectrum. This includes the benign tumor such as schwannoma to most malignant tumors such as glioblastoma multiforme and metastasis. NLR samples were sent in the preoperative period, and after surgery, they were correlated with histopathology. We found that mean NLR in low-grade tumors was 1.68, and in high-grade tumors, it was 3.12 $(p<0.01)$. It is seen that an NLR cutoff value of 2.4 can be used to predict high-grade tumors with high sensitivity and high specificity values.

Tumors depending on their biologic aggressiveness induce inflammatory response, which is reflected as increase in the number of leukocytes and increase in the number of neutrophils in relation to lymphocyte in the peripheral blood..$^{23}$ It is suggested that glioma can synthesize granulocyte colony-stimulating factor (CSF) and granulocyte-macrophage-CSF, which might be responsible for affecting the number of neutrophils. ${ }^{24}$ These factors are also implicated in neutrophil-mediated killing of glioma cells. ${ }^{23}$ It is proposed that in glioblastoma multiforme patients, the increase in neutrophils and decrease in lymphocytes are due to increased margination and apoptosis. Decreased cell-mediated immunity due to loss of lymphocytes may have a role to play in tumor biology. ${ }^{18}$ Multiple studies showed that higher values of NLR are associated with poor survival in patients with metastases and glioblastoma multiforme. ${ }^{9-21}$

The mean NLR of intra-axial tumors $(2.64 \pm 0.91)$ was found to higher than that of extra-axial tumors $(1.68 \pm 0.62)$. This observation was statistically significant, with an NLR cutoff 2; intra-axial can be predicted with a sensitivity of $75 \%$ and a specificity of $61.7 \%$.

NLR value correlates better with the grade of tumor than the location of tumor. High-grade tumors are associated with antigenicity, angioinvasion, breach of blood-brain barrier, and infiltration of dura leading to increased proliferation of peripheral neutrophils. This observation is seen in highgrade intra-axial and also extra-axial tumors.

High-grade meningiomas were found to have a higher mean NLR when compared with Grade I meningiomas. This can be attributed to the biological behavior of these tumors, which probably leads to a higher inflammatory reaction.

\section{Limitations}

This was a study involving 116 patients covering the whole spectrum of brain tumors. Therefore, few tumors were less in numbers; for example, high-grade extra-axial tumors were fewer when compared with other subgroups. This leads to skewed data.

\section{Conclusion}

NLR is an easily available and inexpensive marker of systemic inflammation, which varies across the different types of brain tumors. Mean NLR is higher in high-grade 
tumors than low-grade tumors and a cutoff value of $>2.4$ is suggested. Similarly, mean NLR is higher in intra-axial tumors than extra-axial tumors. Cutoff NLR $>2$ can be used as an adjunct to predict intra-axial brain tumors. Further, large-scale studies are required to fully exploit the potential of this investigation tool.

\section{Funding \\ None.}

\section{Conflict of Interest}

None declared.

\section{References}

1 Templeton AJ, McNamara MG, Šeruga B, et al. Prognostic role of neutrophil-to-lymphocyte ratio in solid tumors: a systematic review and meta-analysis. J Natl Cancer Inst 2014;106(6):dju124

2 Massara M, Persico P, Bonavita O, et al. Neutrophils in gliomas. Front Immunol 2017;8:1349

3 Imtiaz F, Shafique K, Mirza SS, Ayoob Z, Vart P, Rao S. Neutrophil lymphocyte ratio as a measure of systemic inflammation in prevalent chronic diseases in Asian population. Int Arch Med 2012;5(1):2

4 Xiao WK, Chen D, Li SQ, Fu SJ, Peng BG, Liang LJ. Prognostic significance of neutrophil-lymphocyte ratio in hepatocellular carcinoma: a meta-analysis. BMC Cancer 2014;14:117

5 Proctor MJ, McMillan DC, Morrison DS, Fletcher CD, Horgan PG, Clarke SJ. A derived neutrophil to lymphocyte ratio predicts survival in patients with cancer. $\mathrm{Br} \mathrm{J}$ Cancer 2012;107(4):695-699

6 Guthrie GJ, Charles KA, Roxburgh CS, Horgan PG, McMillan DC, Clarke SJ. The systemic inflammation-based neutrophil-lymphocyte ratio: experience in patients with cancer. Crit Rev Oncol Hematol 2013;88(1):218-230

7 Jung MR, Park YK, Jeong O, et al. Elevated preoperative neutrophil to lymphocyte ratio predicts poor survival following resection in late stage gastric cancer. J Surg Oncol 2011;104(5):504-510

8 Kang MH, Go SI, Song HN, et al. The prognostic impact of the neutrophil-to-lymphocyte ratio in patients with small-cell lung cancer. Br J Cancer 2014;111(3):452-460

9 Auezova R, Ryskeldiev N, Doskaliyev A, et al. Association of preoperative levels of selected blood inflammatory markers with prognosis in gliomas. OncoTargets Ther 2016;9:6111-6117

10 Subeikshanan V, Dutt A, Basu D, Tejus MN, Maurya VP, Madhugiri VS. A prospective comparative clinical study of peripheral blood counts and indices in patients with primary brain tumors. J Postgrad Med 2016;62(2):86-90
11 Han S, Zhang C, Li Q, et al. Tumour-infiltrating CD4(+) and $\mathrm{CD} 8(+)$ lymphocytes as predictors of clinical outcome in glioma. Br J Cancer 2014;110(10):2560-2568

12 Bao Y, Yang M, Jin C, et al. Preoperative hematologic inflammatory markers as prognostic factors in patients with glioma. World Neurosurg 2018;119:e710-e716

13 Zhang J, Zhang S, Song Y, et al. Prognostic role of neutrophil lymphocyte ratio in patients with glioma. Oncotarget 2017;8(35):59217-59224

14 Mason M, Maurice C, McNamara MG, et al. Neutrophil-lymphocyte ratio dynamics during concurrent chemo-radiotherapy for glioblastoma is an independent predictor for overall survival. J Neurooncol 2017;132(3):463-471

15 Alexiou GA, Vartholomatos E, Voulgaris S. Prognostic value of neutrophil-to-lymphocyte ratio in patients with glioblastoma. J Neurooncol 2013;115(3):521-522

16 Wang PF, Song HW, Cai HQ et al. Preoperative inflammation markers and IDH mutation status predict glioblastoma patient survival. Oncotarget 2017;8(30):50117-50123

17 Wang J, Xiao W, Chen W, Hu Y. Prognostic significance of preoperative neutrophil-to-lymphocyte ratio and platelet-to-lymphocyte ratio in patients with glioma. EXCLI J 2018;17:505-512

18 Bambury RM, Teo MY, Power DG, et al. The association of pre-treatment neutrophil to lymphocyte ratio with overall survival in patients with glioblastoma multiforme. J Neurooncol 2013;114(1):149-154

19 Han S, Liu Y, Li Q, Li Z, Hou H, Wu A. Pre-treatment neutrophil-to-lymphocyte ratio is associated with neutrophil and T-cell infiltration and predicts clinical outcome in patients with glioblastoma. BMC Cancer 2015;15:617

20 McNamara MG, Lwin Z, Jiang H, et al. Factors impacting survival following second surgery in patients with glioblastoma in the temozolomide treatment era, incorporating neutrophil/ lymphocyte ratio and time to first progression. J Neurooncol 2014;117(1):147-152

21 Mitsuya K, Nakasu Y, Kurakane T, Hayashi N, Harada H, Nozaki K. Elevated preoperative neutrophil-to-lymphocyte ratio as a predictor of worse survival after resection in patients with brain metastasis. J Neurosurg 2017;127(2):433-437

22 Louis DN, Perry A, Reifenberger G, et al. The 2016 World Health Organization classification of tumors of the central nervous system: a summary. Acta Neuropathol 2016;131(6):803-820

23 Fossati G, Ricevuti G, Edwards SW, Walker C, Dalton A, Rossi ML. Neutrophil infiltration into human gliomas. Acta Neuropathol 1999;98(4):349-354

24 Kikuchi T, Nakahara S, Abe T. Granulocyte colony-stimulating factor (G-CSF) production by astrocytoma cells and its effect on tumor growth. J Neurooncol 1996;27(1):31-38 